\title{
Fitorremediação da água de rejeito de dessalinizadores utilizados no oeste potiguar por Pistia statiotes.
}

Hallyson Galdino Marques* (Graduando em Ciência e Tecnologia, Universidade Federal Rural do Semiárido, Caraúbas, RN);

Francisco das Chagas Rodrigue Souza (Graduando em Ciência e Tecnologia, Universidade Federal Rural do Semi-árido, Caraúbas, RN);

Andre Moreira de Oliveira (Docente do curso de Ciência e Tecnologia, Universidade Federal Rural do Semi-árido, Caraúbas, RN);

Daniel Freitas Freire Martins (Docente do curso de Ciência e Tecnologia, Universidade Federal Rural do Semi-árido, Caraúbas, RN);

*E-mail: hallysongaldino@hotmail.com

\section{Resumo:}

Água limpa e de boa qualidade é um produto precioso. No semiárido brasileiro, devido a reconhecida escassez de águas superficiais, um grande desafio é promover o abastecimento de água às famílias rurais. O uso de águas subterrâneas é uma alternativa viável para garantir o acesso dessas comunidades à água. Entretanto, essas fontes hídricas apresentam na maioria dos casos restrições de uso para o consumo humano devido à alta concentração relativa de sais (SOUZA NETO et al., 2010). Desta forma, este trabalho tem como objetivo tratar a água de rejeito do processo de dessalinização, utilizando macrófitas aquáticas da espécie Pistia stratiotes como agente fitorremediador da água do rejeito dos dessalinizadores utilizados no oeste potiguar e verificar possibilidades de uso da água obtida mediante suas características físico-químicas. De acordo com os resultados obtidos, observa-se que ocorreu uma grande redução na concentração de magnésio, saindo de uma concentração inicial de $619,51 \mathrm{mg} \mathrm{L}^{-1}$ para uma concentração $250,42 \mathrm{mg} \mathrm{L}^{-1} \mathrm{em}$ apenas 8 dias. Também houve uma diminuição considerável de cálcio em um curto espaço de tempo, saindo de uma concentração inicial de 474,55 mg L-1 para uma concentração de $372,54 \mathrm{mg} \mathrm{L}^{-1}, 12$ dias após as o início do tratamento.

\section{Palaviras-chave:}

Água salobra; fitorremediação; rejeito da dessalinização; macrófita aquática; Pistia stratiotes. 


\section{INTRODUÇÃO}

Água limpa e de boa qualidade é um produto precioso. No semiárido brasileiro, devido a reconhecida escassez de águas superficiais, um grande desafio é promover o abastecimento de água às famílias rurais. $\mathrm{O}$ uso de águas subterrâneas é uma alternativa viável para garantir o acesso dessas comunidades à água. Entretanto, essas fontes hídricas apresentam na maioria dos casos restrições de uso para o consumo humano devido à alta concentração relativa de sais (SOUZA NETO et al., 2010).

Um tratamento muito utilizado para reduzir a concentração de sais destas águas tem sido a dessalinização por osmose reversa. Entretanto, faz-se necessário que se considerem os riscos ambientais decorrentes desta técnica, isto porque, na dessalinização se gera, além da água potável, uma água residuária (rejeito) altamente salina e com risco de contaminação ambiental elevado (DIAS et al., 2007).

$\mathrm{Na}$ maioria das vezes esse rejeito não recebe qualquer tratamento, mesmo assim, está sendo despejado no solo, propiciando alto acúmulo de sais nas camadas superficiais do terreno, podendo trazer, em curto espaço de tempo, sérios problemas ambientais para as comunidades que se beneficiam da tecnologia de dessalinização (PORTO et al., 2001).

O rejeito quando despejado no solo sem nenhum tratamento, aumenta a concentração dos sais no mesmo. A acumulação de sais, na rizosfera, prejudica o crescimento e desenvolvimento das culturas, provocando um decréscimo de produtividade e, em casos mais severos, pode levar a um colapso da produção agrícola. Isso ocorre em razão da elevação do potencial osmótico da solução do solo, por efeitos tóxicos dos íons específicos e alteração das condições físicas e químicas do solo (LIMA, 1998).

A alta salinidade também causa efeitos sobre as plantas, provocando dificuldades à absorção de água e toxicidade de íons específicos (SILVA et al., 1999) e, ainda, à composição mineral das folhas com interferência nos processos fisiológicos (BOSCO et al., 2009).

Daí surge a necessidade de ser utilizada uma técnica ambientalmente correta, para o tratamento dessa água (rejeito), pois quase sempre os cursos d'água e o solo são os principais meios para sua deposição. De acordo com Raskin e Ensley (2001), a biorremediação é uma tecnologia emergente que visa proporcionar um tratamento barato, suave e seguro, aplicável a ambientes contaminados. A mesma apresenta-se como uma técnica eficaz e de baixo custo à remoção desses sais. Essa técnica consiste no uso de organismos vivos para a remoção ou redução de poluentes no ambiente (GAYLARD et al., 2005). Dentro da biorremediação, a fitorremediação ganha destaque como uma das técnicas mais estudadas. Segundo Jaida e Fulekar (2009) essa técnica consiste basicamente na utilização de plantas na recuperação ou estabilização de ambientes poluídos.

$\mathrm{O}$ uso de macrófitas aquáticas pode oferecer uma função ambientalmente eficaz, sendo um método barato para o tratamento de água contaminada. Isto porque as plantas têm capacidade de absorver elementos essenciais da água através de suas raízes. As vantagens neste tipo de sistema são o baixo custo na implantação, manutenção e elevada eficiência (NUNES, 2012).

Dentre as macrófitas utilizadas na fitorremediação apresenta-se a Pistia stratiotes, conhecida popularmente como alface d'água, pertencente à família Araceae, é uma espécie flutuante com capacidade de rápida multiplicação vegetativa, habilidade para regenerar-se a partir de pequenas porções do talo e independência parcial ou completa das estruturas sexuais de reprodução (MARTINS, 2001).

Desta forma, este trabalho teve como objetivo verificar a viabilidade de utilização de macrófitas aquáticas da espécie Pistia stratiotes como agente fitorremediador da água do rejeito dos dessalinizadores utilizados no oeste potiguar e verificar possibilidades de uso da água obtida mediante suas características físico-químicas. 


\section{MATERIAIS E MÉTODOS}

Os métodos de análises seguidos neste trabalho são de acordo com o Standard Methods of APHA (2005), os quais são sumariamente descritos a seguir.

Inicialmente a água de rejeito dos dessalinizador foi coletada na comunidade Bom Jesus, em Campo Grande-RN, localizada no oeste potiguar, e transportada para a Universidade Federal Rural do Semi-árido, Campus Caraúbas, onde foi armazenada em uma caixas d'água cobertas com um sombrite para minimizar o processo de evaporação. Em seguida uma amostra de água foi levada para o laboratório, onde verificou-se a condutividade e o $\mathrm{pH}$ utilizando um condutivímetro e pHmetro de bancada, respectivamente. Em seguida foram filtradas utilizando papel de filtro qualitativo e feitas as quantificações dos teores de sódio, potássio, cálcio e magnésio presentes na água.

Após a retirada da amostra de água do rejeito para análise inicial, inseriu-se 140 plantas da espécie Pistia stratiotes, anteriormente coletadas em um ambiente natural, lavadas exaustivamente com água da torneira e enxaguadas com água destilada, onde ficaram expostas durante 22 dias. Após 12 dias as plantas foram removidas da caixa d'água e mais 140 novas plantas foram inseridas e permaneceram até o vigésimo segundo dia de experimento.

Com as plantas já inseridas na caixa fez-se o acompanhamento com intervalo entre 3 e 4 dias de uma coleta para outra, para acompanhar a eficiência das plantas na remoção dos sais dispostos na água.

Para que a concentração de sais não fosse afetada pelo fator evaporação, antes de cada coleta, completava-se com água destilada o nível da caixa até o ponto demarcado na primeira coleta seguido de intensa agitação para garantir a homogeneização da solução.

Em todas as coletas, a condutividade e $\mathrm{pH}$ foram determinados como citado anteriormente, a quantificação de sódio e potássio na água foi realizada em um fotômetro de chama, modelo 7000 da marca TECNOW, enquanto que a análise de cálcio e magnésio foi feita por titulação complexométrica utilizando EDTA como agente complexante. Para a determinação do teor de magnésio, foi necessária a determinação da dureza total também.

A seguir apresenta-se uma descrição mais detalhada dos procedimentos de análise realizados.

\subsection{Condutividade}

Foi determinada por método eletrométrico, utilizando-se um condutivímetro manual, marca HANNA instruments, modelo DIST WP, recentemente calibrado com solução padrão 146,9 $\mu \mathrm{S} / \mathrm{cm}$.

\section{2. $\mathbf{p H}$}

$\mathrm{O} \mathrm{pH}$ foi determinado por método eletrométrico, utilizando-se um pHmetro marca TECNAL, modelo Tec-3MP recentemente calibrado com soluções padrões apropriadas.

\subsection{Dureza Total}

Determinada através de titulação complexométrica, utilizando solução padrão de EDTA 0,011 mol/L como titulante, indicador negro de eriocromo T, solução tampão de $\mathrm{NH}_{4} \mathrm{Cl} / \mathrm{NH}_{4} \mathrm{OH}$ para manutenção do $\mathrm{pH}$ entre 10 e 11.

Anota-se o volume de EDTA gasto na titulação e a dureza total é calculada através da Equação 1 e 2. DT $(\mathrm{ppm})=\mathrm{V}_{\text {gasto EDTA }} \times$ Fator $\mathrm{CaCO}_{3} \times 1000 / \mathrm{V}_{\text {amostra }}$ Equação 1

Onde:

Fator $\mathrm{CaCO}_{3}=$ Molaridade EDTA x $\mathrm{MMCaCO}_{3}$

Equação 2

$\mathrm{V}_{\text {gasto EDTA }}=$ volume $(\mathrm{em} \mathrm{mL})$ de EDTA gasto na titulação.

$\mathrm{V}_{\text {amostra }}=$ Volume da amostra usado $(\mathrm{em} \mathrm{mL})$.

\subsection{Cálcio}

O cálcio é determinado por titulação complexométrica utilizando como titulante solução padrão de EDTA $0,011 \mathrm{~mol} / \mathrm{L}$, solução $\mathrm{NaOH} 2 \mathrm{~N}$ para elevar o pH acima de 12, e o indicador azul de eriocromo $\mathrm{R}$ ou Calcon.

Anota-se o volume gasto de EDTA na titulação e calcula-se a quantidade de cálcio através da Equação 3 e 4.

$\mathrm{Ca}^{2+}(\mathrm{ppm})=\mathrm{V}_{\text {gasto EDTA }} \times$ Fator $\mathrm{Ca}^{2+} \times 1000 / \mathrm{V}_{\text {amostra }}$ 
Onde:

Fator $\mathrm{Ca}^{2+}=$ Molaridade EDTA x $\mathrm{MMCa}^{2+}$

Equação 4

$\mathrm{V}_{\text {gasto EDTA }}=$ volume $(\mathrm{em} \mathrm{mL})$ de EDTA gasto na titulação.

$\mathrm{V}_{\text {amostra }}=$ Volume da amostra usado $(\mathrm{em} \mathrm{mL})$.

\subsection{Magnésio}

O magnésio é determinado através da diferença do volume gasto de EDTA na dureza total pelo volume gasto de EDTA na determinação do cálcio, sendo calculado sua quantidade em ppm pela Equação 5 e 6.

$\mathrm{Mg}^{2+}(\mathrm{ppm})=\left(\mathrm{V}_{\text {gasto EDTA na DT }}-\mathrm{V}_{\text {gasto EDTA no Ca2+ }}\right) \times$ Fator $\mathrm{Mg}^{2+}$ x $1000 / \mathrm{V}_{\text {amostra }}$

Equação 5

Onde:

Fator $\mathrm{Mg}^{2+}=$ Molaridade do EDTA x $\mathrm{MMMg}^{2+}$

Equação 6

$\mathrm{V}_{\text {gasto EDTA }}$ na $\mathrm{DT}=$ volume $(\mathrm{em} \mathrm{mL})$ de EDTA gasto na titulação da dureza total

$\mathrm{V}_{\text {gasto EDTA }}$ no $\mathrm{Ca}^{2+}=$ volume $(\mathrm{em} \mathrm{mL})$ de EDTA gasto na titulação do cálcio

$\mathrm{V}_{\text {amostra }}=$ Volume da amostra usado $(\mathrm{em} \mathrm{mL})$.

\section{RESULTADOS E DISCUSSÃO}

Os resultados iniciais para condutividade e $\mathrm{pH}$, da água salobra estão de acordo com os resultados apresentados por (Anders et al, 2015), que em seu trabalho analisou as propriedades Físico-Química da água salobra em 10 comunidades diferentes no município de Mossoró-RN, e obteve valores entre 7,0 e 8,1 com relação ao $\mathrm{pH}$, e valores entre $0,8 \mathrm{mS} / \mathrm{cm}$ a $10,5 \mathrm{mS} / \mathrm{cm}$ para a condutividade. Já para as demais propriedades analisadas, os valores apresentados pelo mesmo autor estão bem abaixo dos valores obtidos neste trabalho, isso se justifica pelo fato de água de diferentes comunidades apresentarem teores de sais diferentes.

De acordo com os resultados apresentados no Gráfico 1, pode-se observar que os valores de condutividade tiveram uma pequena variação. $\mathrm{O}$ valor inicial era $7,53 \mathrm{mS} / \mathrm{cm}$ na primeira coleta e após o tratamento passou a ser de $6,75 \mathrm{mS} / \mathrm{cm}$. A redução da condutividade elétrica foi devida à absorção dos íons em estudo pelas plantas, pois os nutrientes não foram repostos durante o ciclo da cultura (DIDONE, 2001).

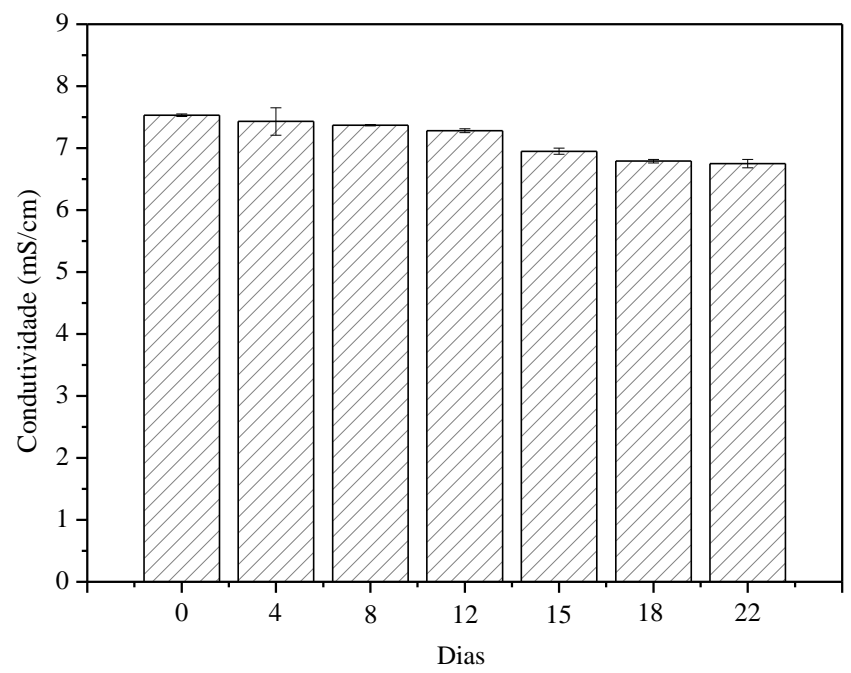

Gráfico 1: Valores Médios e desvio padrão da Condutividade.

O Gráfico 2 apresenta os valores médios e desvio padrão do $\mathrm{pH}$ das sete analises realizadas. Observa-se que houve uma pequena variação, alcançando-se o menor valor na quarta coleta, e estando todos os demais valores bem próximos da neutralidade, estando dentro dos limites permitidos na Resolução CONAMA N ${ }^{\circ}$ 020/1986, para água salobra, pH entre 6,5 a 8,5 podendo ser utilizada para 
recreação de contato primário, à proteção das comunidades aquáticas e à criação natural ou/e intensiva de espécies destinadas à alimentação humana.

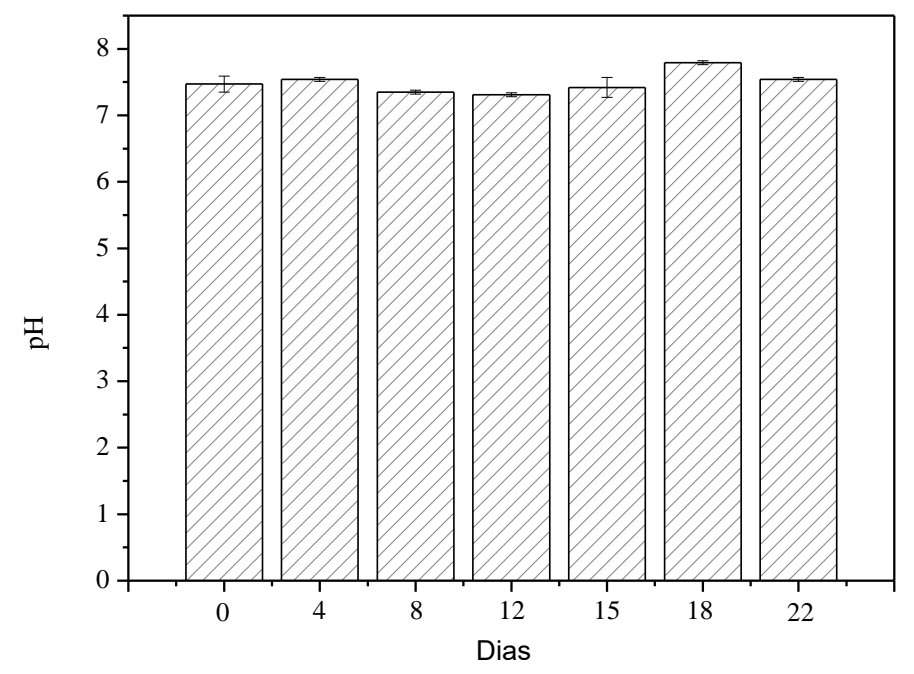

Gráfico 2: Valores médios e desvio padrão do pH das amostras.

No Gráfico3 estão dispostos os valores médios e desvio padrão à concentração de sódio dispostos na água, analisando-se gráfico, observa-se dois comportamentos. Primeiro que houve diminuição da concentração inicial até o $12^{\circ}$ dia, tempo esse que permaneceram as primeiras plantas. $\mathrm{O}$ segundo comportamento, foi o aumento da concentração de $\mathrm{Na}$ a partir da quarta análise. Isso se justifica, provavelmente, pelo fato que as plantas colocadas após esse período estarem em um ciclo de vida mais avançado (plantas maiores, no final do ciclo de vida) e, com isso, elas tendem a lançar para o meio alguns elementos absorvidos durante seu ciclo de vida. Este fato pode ser evitado em trabalhos posteriores fazendo-se a utilização de plantas jovens (no início do seu ciclo de vida).

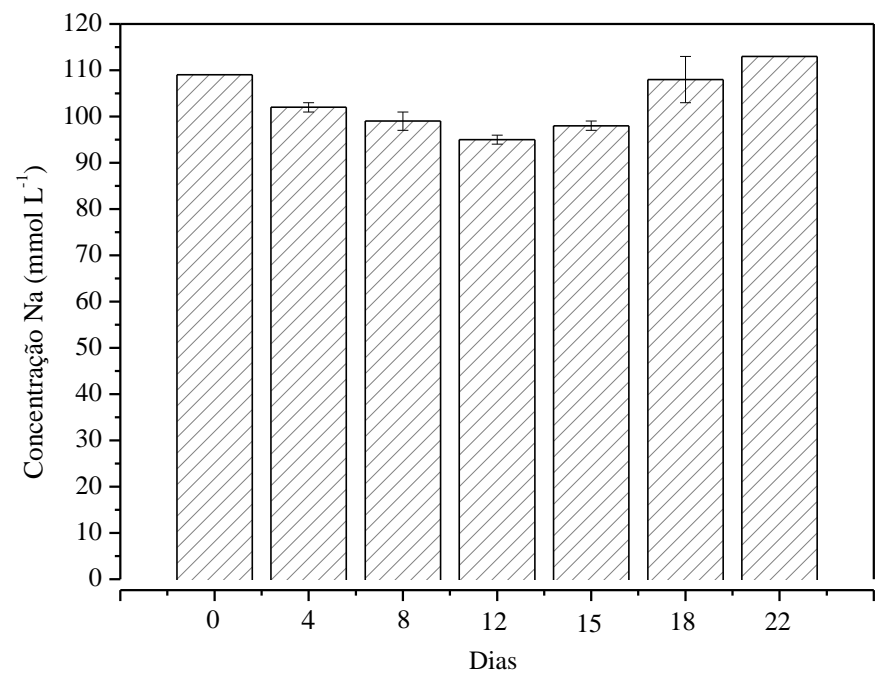

Gráfico 3: Valores médios e desvio padrão da concentração de sódio.

Trabalhos apresentados na literatura mostram que plantas mais jovens apresentam maior capacidade de absorção de nutrientes do meio e com o decorrer do seu ciclo de vida, essa capacidade tende a diminuir significativamente (MARTINS et al., 2011). 
No Gráfico 4 estão dispostos os valores médios e desvio padrão com relação a concentração de potássio. Como pode-se observar, não houve alteração na concentração de $\mathrm{K}$, isso por que o sódio, apesar de ser um micronutriente, e ser necessário em menor quantidade que o potássio (macronutriente), está em quantidade muito superior a este último na água. Além disso, o sódio tem mesma carga e menor raio iônico que o potássio, o que facilita a absorção pelas raízes das plantas.

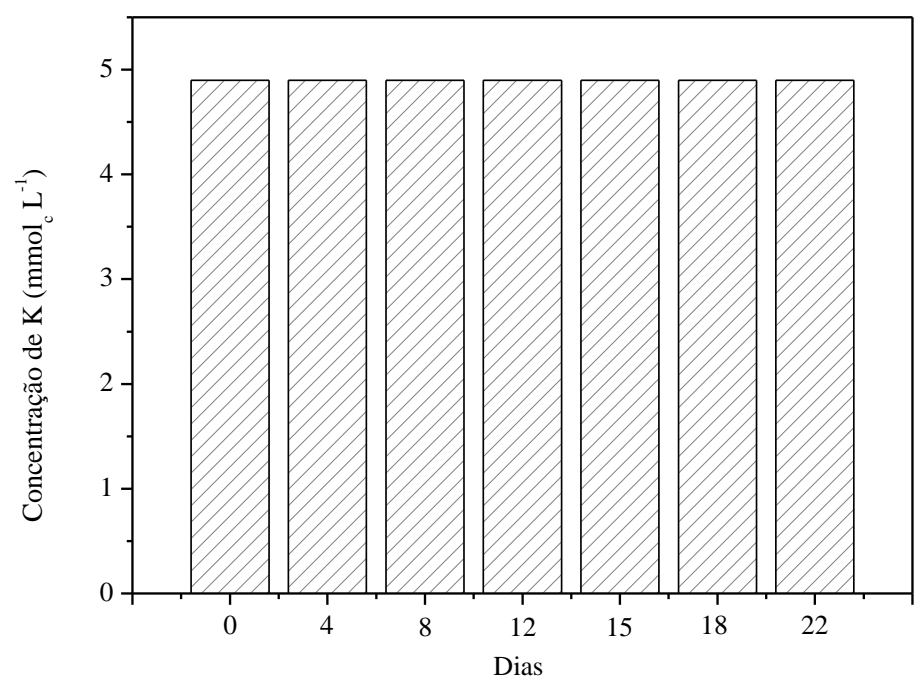

Gráfico 4: Valores médios e desvio padrão da concentração de potássio.

De acordo com os resultados apresentados no gráfico 5, observa-se que ocorreu uma grande redução na concentração de magnésio, saído de uma concentração inicial de $619,51 \mathrm{mg} \mathrm{L}^{-1}$ para uma concentração $250,42 \mathrm{mg} \mathrm{L}^{-1}$ com apenas 8 dias. Observa-se também que após 12 dias de tratamento, período em que as plantas foram trocadas, a concentração de $\mathrm{Mg}$ manteve-se praticamente constante, apenas com um pouco de acréscimo. Resultado esse muito expressivo, com uma redução de 369,09 $\mathrm{mg} \mathrm{L}^{-1} \mathrm{em}$ apenas uma semana.

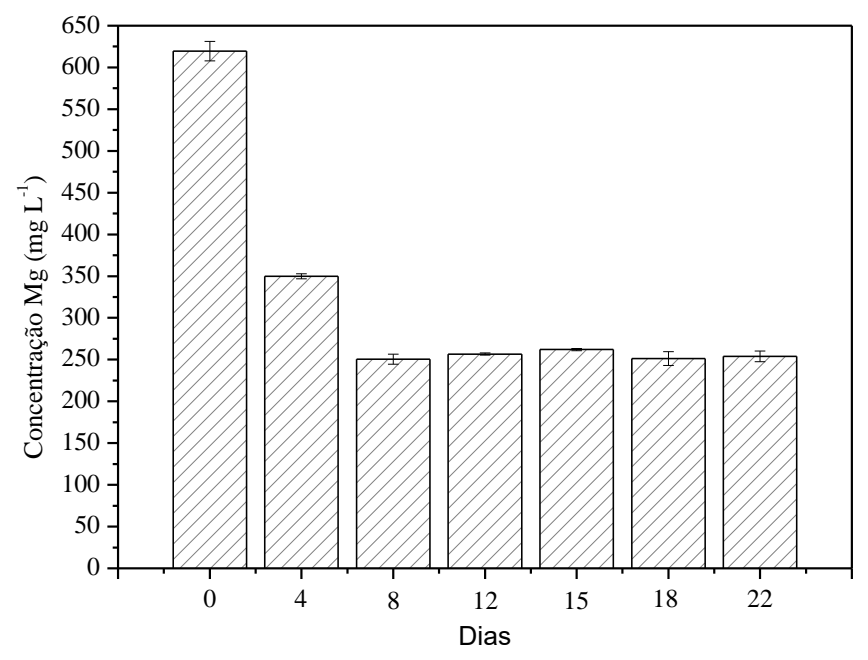

Gráfico 5: Valores médios e desvio padrão para a concentração de magnésio.

No Gráfico 6 estão dispostos os valores médios e desvio padrão com relação a concentração do cálcio existente na água salobra. Ao analisar o gráfico, observa-se que houve uma diminuição considerável em um curto espaço de tempo. Saindo de uma concentração inicial de $474,55 \mathrm{mg} \mathrm{L}^{-1}$ para 
uma concentração de $372,54 \mathrm{mg} \mathrm{L}^{-1}$ após 12 dias. Assim como para o magnésio após a o $12^{\circ}$ dia, os valores mantiveram-se quase constantes.

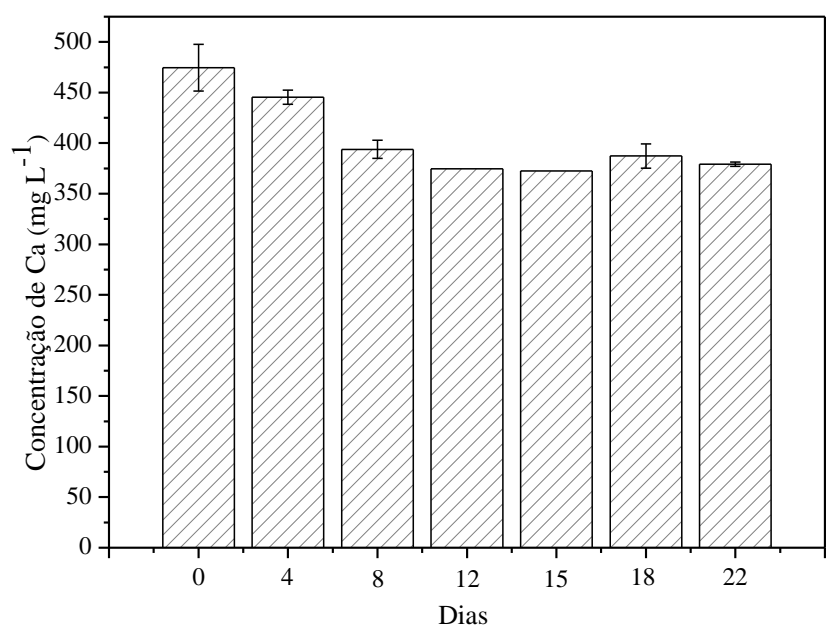

Gráfico 6: Valores médios e desvio padrão para a concentração de cálcio.

\section{CONCLUSÕES}

A partir da análise dos resultados obtidos neste trabalho, chegou-se as seguintes conclusões:

a. Pode-se constatar que houve uma diminuição significativa para os teores de cálcio, magnésio e sódio. Portanto, a água que era imprópria para uso e descartada no ambiente, com um tratamento simples e barato como a fitorremediação, pode ter a sua utilização destinada para algum fim;

b. De forma geral, pôde-se então constatar também que os melhores resultados para absorção dos nutrientes ocorreram na quarta coleta, período em que foram utilizadas as menores plantas;

c. É necessário um estudo mais aprofundado a respeito do processo utilizado a fim de identificar condições as quais se consiga remover o máximo de sais possíveis, e assim, obter-se uma água mais nobre e que possa ser destinada para um fim específico.

\section{Desalination plants of waste water used in phytoremediation Natal west by Pisria Statiotes.}

Abstract: Clean and good quality water is a precious commodity. In the Brazilian semiarid, recognized due to shortage of surface water, a major challenge is to promote the supply of water to rural families. The use of groundwater is a viable alternative to ensure access of these communities to water. However, these water sources present in most cases use restrictions for human consumption due to the high relative concentration of salts Thus, this paper aims to treat waste water from the desalination process using aquatic weeds of stratiotes Pistia species as phytoremediation agent reject water from desalination plants used in Potiguar West and verify possibilities of using water obtained by their physical-chemical characteristics. According to the results, it was observed that there was a large decrease in the magnesium concentration, out of an initial concentration of $619.51 \mathrm{mg} \mathrm{L}-1$ for a $250.42 \mathrm{mg} \mathrm{L}-1$ concentration in only 8 days. There was also a considerable decrease of calcium in a 
short time, leaving an initial concentration of $474.55 \mathrm{mg} \cdot \mathrm{L}^{-1}$ to a concentration of $372.54 \mathrm{mg} \cdot \mathrm{L}^{-1}, 12$ days after initiation of treatment.

Keywords: Brackish water; phytoremediation; reject desalination; macrophyte; Pistia stratiotes.

\section{REFERÊNCIAS BIBLIOGRÁFICAS}

AMERICAN PUBLIC HEALTH ASSOCIATION. Standard methods for the examination of water and wastewater. 18 th ed. Washington: APHA. 2005.

BOSCO, M. R. O; OLIVEIRA, A. B; HERNANDEZ, F. F. F; LACERDA, C. F; Influência do estresse salino na composição mineral da berinjela. Revista Ciência Agronômica, 40:157-164.

CONAMA No 020/1986. Resolução nº 20, de 18 de junho de 1986 (Diário Oficial, seção 1, 30/07/1986, p. $11356-11360)$.

COSTA, P. C; DIDONE, E. B; SESSO, T. M; CAÑIZARES, K. A. L; GOTO, R. Condutividade elétrica da solução nutritiva e produção da alface em hidroponia. Scientia Agricola, v.58, n.3, p.595-597, 2001.

DIAS, N. S; ANDERS, C. R; RANGEL. M; SILVA J. F; SOUZA, A. C; PORTELA, J. C. CARACTERIZAÇÃO FÍSICO-QUÍMICA DA ÁGUA DE POÇO, DO REJEITO DA DESSALINIZAÇÃO E DA ÁGUA DESSALINIZADA, Fortaleza, 2015.

DIAS, N. S. et al. Salinização do solo por aplicação de fertilizantes em ambiente protegido. Irriga, Botucatu, v. 12, n. 1, p. 135-143, 2007.

DIAS, N. S.; JALES, A. G. O.; SOUSA NETO, O.N.; GONZAGA, M.I.S.; QUEIROZ, I. S. R. Q.; PORTO, M. A. F. Uso de rejeito da dessalinização na solução nutritiva da alface cultivada em fibra de coco. Revista Ceres, Viçosa-MG, v.58, p.407-410, 2011.

GAYLARDE, C. C.; BELLINASO, M. L.; MANFIO, G. P. Biorremediação: Aspectos biológicos e técnicos da biorremediação de xenobióticos. Biotecnologia, Ciência e Desenvolvimento, n. 34, Jan/Jun, 2005.

JADIA, C. D.; FULEKAR, M. H. Phytoremediation of heave metals: Recent techniques. African Journal of Biotechnology, v.8, n. 6, p. 921-928, 2009.

Martins , D., ED VELINI , E. Negrisoli , GR Tofoli . 2002. O controle químico de Pistia stratiotes , Eichhornia crassipes e Salvinia molesta em reservatórios . Planta Daninh. 20 : 83-88

MARTINS, Daniel Freitas Freire; de Fátima Vitória de Moura, Maria ; Bezerra Loiola, Maria Iracema ; Di Souza, Luiz ; Barbosa e Silva, Káthia Maria ; Francismar de Medeiros, José . Temporal and physiological influence of the absorption of nutrients and toxic elements by Eichhornia crassipes. Journal of Environmental Monitoring (Print), p. 274-279, 2011.

NUNES, S. S.; avaliação da inoculsção de fungos na rizosfera da Pistia stratiotes visando a sua utilização no tratamento de efluentes urbanos. Santa Cruz do Sul, 2012.

PORTO, E. R; AMORIM, M. C. C; SILVA JUNIOR, L. G. A; Uso do rejeito da dessalinização de água salobra para irrigação da ervasal (Atriplex nummularia). Revista Brasileira de Engenharia Agrícola e Ambiental, 5:111-114.

RASKIN, I.; ENSLEY, B. Phytoremediation of Toxic Metals. Using Plants to Clean Up the Environment. Plant Science, v. 160, p. $1073-1075,2001$.

SILVA, E. F. F; DUARTE, S. N; FURLAN, R. A; FERREIRA, Y. R. P; MIRANDA, J. H; Utilização de águas com diferentes níveis de salinidade na irrigação da alface. Revista de Horticultura Brasileira, 1999. 